\title{
The contact process with semi-infected state on the complete graph
}

\author{
Xiaofeng Xue * \\ Beijing Jiaotong University
}

\begin{abstract}
In this paper we are concerned with the contact process with semi-infected state on the complete graph $C_{n}$ with $n$ vertices. In our model, each vertex is in one of three states that 'healthy', 'semi-infected' or 'wholly-infected'. Only wholly-infected vertices can infect others. A healthy vertex becomes semi-infected when being infected while a semi-infected vertex becomes wholly-infected when being further infected. Each (semi- and wholly-) infected vertex becomes healthy at constant rate. Our main result shows the phase transition for the time wholly-infected vertices wait for to die out. Conditioned on all the vertices are wholly-infected when $t=0$, we show that wholly-infected vertices survive for $\exp \{O(n)\}$ units of time when the infection rate $\lambda>4$ while die out in $O(\log n)$ units of time when $\lambda<4$.
\end{abstract}

Keywords: Contact process, semi-infected, complete graph, phase transition.

\section{Introduction}

In this paper we are concerned with the contact process with semi-infected state on the complete graph. A complete graph is a finite graph such that for any two vertices there is an edge connecting them. For later use, for integer $n \geq 1$, we denote by $C_{n}$ the complete graph with $n$ vertices and denote by $\{1,2, \ldots, n\}$ the vertices set of $C_{n}$.

The contact process with semi-infected state on $C_{n}$ is a continuous-time Markov process with state space $\{0,1,2\}^{C_{n}}$, i. e. at each vertex there is a spin taking value from $\{0,1,2\}$. For any configuration $\eta \in\{0,1,2\}^{C_{n}}$ and $1 \leq i \leq n$, we denote by $\eta(i)$ the value of the spin at the vertex $i$. For any $t \geq 0$, we denote by $\eta_{t}$ the configuration of our process at moment $t$. For $\eta \in\{0,1,2\}^{C_{n}}, 1 \leq i \leq n$ and $0 \leq l \leq 2$, we define $\eta^{i, l} \in\{0,1,2\}^{C_{n}}$ as follows.

$$
\eta^{i, l}(j)= \begin{cases}\eta(j), & \text { if } j \neq i, \\ l, & \text { if } j=i .\end{cases}
$$

The generator $\Omega$ of $\left\{\eta_{t}\right\}_{t \geq 0}$ has the form

$$
\Omega f(\eta)=\sum_{1 \leq i \leq n} \sum_{l=0,1,2} H(\eta, i, l)\left[f\left(\eta^{i, l}\right)-f(\eta)\right]
$$

\footnotetext{
*E-mail: xfxue@bjtu.edu.cn Address: School of Science, Beijing Jiaotong University, Beijing 100044, China.
} 
for any continuous function $f$ on $\{0,1,2\}^{C_{n}}$. That is to say, at each moment that the configuration of the process jumps, only one spin changes value. Conditioned on the current configuration $\eta$, the process jumps to $\eta^{i, l}$ at rate $H(\eta, i, l) . H(\eta, i, l)$ is defined as

$$
H(\eta, i, l)= \begin{cases}1 & \text { if } l=0 \text { and } \eta(i) \neq 0, \\ \frac{\lambda}{n}|\{j: \eta(j)=2\}| & \text { if } l=2 \text { and } \eta(i)=1, \\ \frac{\lambda}{n}|\{j: \eta(j)=2\}| & \text { if } l=1 \text { and } \eta(i)=0 \\ 0 & \text { else, }\end{cases}
$$

where $\lambda$ is a positive constant called the infection rate and $|A|$ is the cardinality of the set A.

Intuitively, the process describes the spread of an epidemic, where each individual is in one of the three states that healthy, semi-infected or wholly-infected. For details, vertices in state 0 are healthy while vertices in state 1 are semi-infected and vertices in state 2 are wholly-infected. Each infected vertex, no matter semi- or wholly-, waits for an exponential time with rate 1 to become healthy. Wholly-infected vertices have the ability to infect others. A healthy vertex is infected at rate proportional to the number of wholly-infected vertices and becomes a semi-infected vertex when being infected. A semi-infected vertex is further infected at rate proportional to the number of wholly-infected vertices and becomes a wholly-infected vertex when being infected.

According to the spatial homogeneity of the process $\left\{\eta_{t}\right\}_{t \geq 0}$, we only care about the numbers of vertices in state 1 and 2 . Hence we define

$$
B_{t}=\left|\left\{i: \eta_{t}(i)=2\right\}\right| \text { and } G_{t}=\left|\left\{i: \eta_{t}(i)=1\right\}\right|
$$

for any $t \geq 0$. According to the generator of $\left\{\eta_{t}\right\}_{t \geq 0}$ given in Equation (1.1) and the definition of $H(\eta, i, l)$, the transition rates function of $\left\{\left(B_{t}, G_{t}\right)\right\}_{t \geq 0}$ is given by

$$
(B, G) \text { jumps to } \begin{cases}(B-1, G) & \text { at rate } B, \\ (B, G-1) & \text { at rate } G, \\ (B+1, G-1) & \text { at rate } \frac{\lambda}{n} B G, \\ (B, G+1) & \text { at rate } \frac{\lambda}{n} B(n-B-G) .\end{cases}
$$

The process $\left\{\eta_{t}\right\}_{t \geq 0}$ is an extension of the classic contact process introduced in $[6$ by Harris, where vertices are distinguished as healthy ones and infected ones such that a healthy one is infected at rate proportional to the number of infected neighbors while an infected one becomes healthy at rate one. For a detailed survey of the study of the classic contact process, see Chapter 6 of [7] and Part one of [8.

In [9, Peterson studies the contact process on the complete graph with vertex-dependent infection rates, containing the classic contact process as a case. It is shown in [9] that there is a critical value $\lambda_{c}$ of the infection rate $\lambda$ such that when $\lambda<\lambda_{c}$ the process dies out before $O(\log n)$ units of time with high probability while when $\lambda>\lambda_{c}$ the process survives for $\exp \{O(n)\}$ units of time with high probability. We are inspired by [9 a lot. Our main result shows that similar phase transition with that in 9 . occurs for the contact process with semi-infected state. The precise value of the critical infection rate is also given. For mathematical details, see Section 2 


\section{Main result}

In this section we give the main result of this paper. We care about the first moment that there is no vertex in state 2 , hence we define

$$
\tau=\inf \left\{t: B_{t}=0\right\} .
$$

Since vertices in state 1 can not infect others, after the moment $\tau$, the epidemic dies out in $\log n$ units of time with high probability, which depends on the fact that the maximum of $n$ independent exponential times with rate one is with order $\log n$ with high probability. As a result, whether the epidemic survives for a long time, for instance $\exp \{O(n)\}$ units of time, depends on $\tau$.

For each $n \geq 1$ and any $\lambda>0$, we denote by $P_{\lambda, n}$ the contact process with semi-infected state on $C_{n}$ with infection rate $\lambda$. We write $P_{\lambda, n}$ as $P_{\lambda, n}^{B, G}$ when $\left(B_{0}, G_{0}\right)=(B, G)$. For given $b, g \in[0,1]$, we write $(B, G)=(n b, n g)$ when $(B, G)=(\lfloor n b\rfloor,\lfloor n g\rfloor)$ for simplicity. We have the following main result which shows phase transition for $\tau$.

Theorem 2.1. When $\lambda>4$, there exists a constant $C=C(\lambda)>0$ such that

$$
\lim _{n \rightarrow+\infty} P_{\lambda, n}^{n, 0}\left(\tau>e^{C n}\right)=1
$$

while when $\lambda<4$,

$$
\lim _{n \rightarrow+\infty} P_{\lambda, n}^{n, 0}(\tau<(1+\theta) \log n)=1 .
$$

for any $\theta>0$.

According to Theorem 2.1 conditioned on all the vertices are wholly-infected at $t=0$, the wholly-infected vertices survive for $\exp \{O(n)\}$ units of time when $\lambda>4$ while die out in $O(\log n)$ units of time when $\lambda<4$.

We are inspired by former references about the contact process on finite sets to prove Theorem 2.1. According to the main results in [9, the classic contact process on the complete graph $C_{n}$ survives for $\exp \{O(n)\}$ units of time when $\lambda>1$ while dies out in $O(\log n)$ units of time when $\lambda<1$. Foxall, Edwards and van den Driessche introduce the contact process on the complete graph incorporating monogamous dynamic partnerships in [5], where similar phase transition with that in 9 is shown and the precise value of the critical infection rate is given. Durrett and coworkers study the contact process on the finite lattice $[-N, N]^{d}$ in 1, 2, 3. One of their main results is that the process survives for $\exp \left\{O\left(N^{d}\right)\right\}$ units of time when $\lambda>\lambda_{c}(d)$ while dies out in $O(\log N)$ units of time when $\lambda<\lambda_{c}(d)$, where $\lambda_{c}(d)$ is the minimum of the infection rates of the contact process on $\mathbb{Z}^{d}$ with which the process survives forever with positive probability.

The proof of Theorem 2.1 is divided into three sections. In Section 3, we introduce an two-dimensional ODE

$$
\left\{\begin{array}{l}
\frac{d}{d t} b_{t}=F_{1}\left(b_{t}, g_{t}\right) \\
\frac{d}{d t} g_{t}=F_{2}\left(b_{t}, g_{t}\right)
\end{array}\right.
$$

and show that the solution $\left(b_{t}, g_{t}\right)$ to this ODE is the mean field limit of $\left(\frac{B_{t}}{n}, \frac{G_{t}}{n}\right)$ for $t$ in any compact area as $n$ grows to infinity. In Section 4, we give the proof of Equation (2.1). The proof relies heavily on the fact that when $\lambda>4$ there exists $(b, g)$ such that $F_{1}(b, g)>0$ 
and $F_{2}(b, g)>0$. In Section 5 , we give the proof of Equation (2.2). The proof relies heavily on the fact that $(0,0)$ is the unique equilibrium state of the ODE when $\lambda<4$.

For later use, at the end of this section we give a lemma which shows that the contact process with semi-infected state is monotonic under a specific partial order on $\mathbb{Z}^{2}$. For $\left(B_{1}, G_{1}\right),\left(B_{2}, G_{2}\right) \in \mathbb{Z}^{2}$, we write $\left(B_{1}, G_{1}\right) \succeq\left(B_{2}, G_{2}\right)$ when and only when $B_{1} \geq B_{2}$ and $B_{1}+G_{1} \geq B_{2}+G_{2}$. It is easy to check that $\succeq$ is a partial order on $\mathbb{Z}^{2}$. We write $\left(B_{2}, G_{2}\right) \preceq\left(B_{1}, G_{1}\right)$ when $\left(B_{1}, G_{1}\right) \succeq\left(B_{2}, G_{2}\right)$. We have the following lemma.

Lemma 2.2. For $j=1,2$, if $\left\{\left(B_{t}^{j}, G_{t}^{j}\right)\right\}_{t \geq 0}$ is the contact process with semi-infected state with initial state $\left(B_{0}^{j}, G_{0}^{j}\right)$ and $\left(B_{0}^{1}, G_{0}^{1}\right) \succeq\left(B_{0}^{2}, G_{0}^{2}\right)$, then $\left(B_{t}^{1}, G_{t}^{1}\right) \succeq\left(B_{t}^{2}, G_{t}^{2}\right)$ for any $t \geq 0$ in the sense of coupling.

Proof. Conditioned on $\left(B^{1}, G^{1}\right) \succeq\left(B^{2}, G^{2}\right)$, we couple the two processes as follows.

$$
\begin{aligned}
& \left(\left(B^{1}, G^{1}\right),\left(B^{2}, G^{2}\right)\right) \rightarrow \\
& \begin{cases}\left(\left(B^{1}-1, G^{1}\right),\left(B^{2}-1, G^{2}\right)\right) & \text { at rate } B^{2}, \\
\left.\left(B^{1}-1, G^{1}\right),\left(B^{2}, G^{2}\right)\right) & \text { at rate } B^{1}-B^{2}, \\
\left.\left(B^{1}, G^{1}-1\right),\left(B^{2}, G^{2}-1\right)\right) & \text { at rate } \min \left\{G^{1}, G^{2}\right\}, \\
\left.\left(B^{1}, G^{1}-1\right),\left(B^{2}, G^{2}\right)\right) & \text { at rate } G^{1}-\min \left\{G^{1}, G^{2}\right\}, \\
\left.\left(B^{1}, G^{1}\right),\left(B^{2}, G^{2}-1\right)\right) & \text { at rate } G^{2}-\min \left\{G^{1}, G^{2}\right\}, \\
\left.\left(B^{1}+1, G^{1}-1\right),\left(B^{2}+1, G^{2}-1\right)\right) & \text { at rate } \frac{\lambda}{n} \min \left\{B^{1} G^{1}, B^{2} G^{2}\right\}, \\
\left.\left(B^{1}+1, G^{1}-1\right),\left(B^{2}, G^{2}\right)\right) & \text { at rate } \frac{\lambda}{n}\left(B^{1} G^{1}-\min \left\{B^{1} G^{1}, B^{2} G^{2}\right\}\right), \\
\left.\left(B^{1}, G^{1}\right),\left(B^{2}+1, G^{2}-1\right)\right) & \text { at rate } \frac{\lambda}{n}\left(B^{2} G^{2}-\min \left\{B^{1} G^{1}, B^{2} G^{2}\right\}\right), \\
\left.\left(B^{1}, G^{1}+1\right),\left(B^{2}, G^{2}+1\right)\right) & \text { at rate } a, \\
\left.\left(B^{1}, G^{1}+1\right),\left(B^{2}, G^{2}\right)\right) & \text { at rate } \frac{\lambda}{n} B^{1}\left(n-B^{1}-G^{1}\right)-a, \\
\left.\left(B^{1}, G^{1}\right),\left(B^{2}, G^{2}+1\right)\right) & \text { at rate } \frac{\lambda}{n} B^{2}\left(n-B^{2}-G^{2}\right)-a,\end{cases}
\end{aligned}
$$

where

$$
a=\frac{\lambda}{n} \min \left\{B^{1}\left(n-B^{1}-G^{1}\right), B^{2}\left(n-B^{2}-G^{2}\right)\right\} .
$$

By direct calculation, it is easy to check that the Markov process with the above transition rates function is a coupling of $\left(B_{t}^{1}, G_{t}^{1}\right)$ and $\left(B_{t}^{2}, G_{t}^{2}\right)$ while all the state transitions hold the property that $\left(B^{1}, G^{1}\right) \succeq\left(B^{2}, G^{2}\right)$.

\section{$3 \quad$ Mean field limit}

In this section we introduce an ODE, the solution to which is the mean field limit of $\left(\frac{B_{t}}{n}, \frac{G_{t}}{n}\right)$ as $n$ grows to infinity. For later use, for any $x=(b, g) \in \mathbb{R}^{2}$, we use $\|x\|_{1}$ to denote $|b|+|g|$, which is the $l_{1}$ norm of $x=(b, g)$. 

$[0,1]$.

We consider the following two-dimensional ODE with initial condition $\left(b_{0}, g_{0}\right) \in[0,1] \times$

$$
\left\{\begin{array}{l}
\frac{d}{d t} b_{t}=-b_{t}+\lambda b_{t} g_{t}, \\
\frac{d}{d t} g_{t}=-g_{t}-\lambda b_{t} g_{t}+\lambda b_{t}\left(1-b_{t}-g_{t}\right),
\end{array}\right.
$$

where $\lambda>0$. For simplicity, we write the above ODE as

$$
\frac{d}{d t}\left(b_{t}, g_{t}\right)=F\left(b_{t}, g_{t}\right)
$$

where $F=\left(F_{1}, F_{2}\right)$ such that $F_{1}(b, g)=-b+\lambda b g$ and $F_{2}(b, g)=-g-\lambda b g+\lambda b(1-b-g)$.

Later we will show that $\frac{1}{n}\left(B_{t}, G_{t}\right)$ converges to the solution $\left(b_{t}, g_{t}\right)$ to ODE (3.1) in probability. By direct calculation, it is easy to check that

$$
F(b, g)=(0,0)
$$

has the unique solution $(0,0)$ when $\lambda<4$ while has two solutions in $(0,1) \times(0,1)$ when $\lambda>4$, which intuitively explains why the critical value of our process is 4 .

We define $\Lambda=\{(b, g): b \geq 0, g \geq 0, b+g \leq 1\} \subseteq \mathbb{R}^{2}$, then we have the following lemma.

Lemma 3.1. The solution $\left(b_{t}, g_{t}\right)$ to $O D E$ (3.1) with initial condition $\left(b_{0}, g_{0}\right) \in \Lambda$ exists for $t \in[0,+\infty)$ and is unique. Furthermore, for any $t \geq 0,\left(b_{t}, g_{t}\right) \in \Lambda$.

Proof. It is easy to check that $F$ satisfies the local Lipschitz condition under the norm $\|\cdot\|_{1}$, according to which the uniqueness of the solution holds. On the boundary of $\Lambda$, it is easy to check that any vector of the vector-field of ODE (3.1) points to the inner of $\Lambda$, hence the solution is absorbed in the area $\Lambda$. On the area $\Lambda$, it is easy to check that $F$ satisfies the global Lipschitz condition, hence the solution exists for $t \in[0,+\infty)$.

The next lemma shows that $\left(\frac{B_{t}}{n}, \frac{G_{t}}{n}\right)$ converges to the solution $\left(b_{t}, g_{t}\right)$ to the ODE (3.1) in probability as $n$ grows to infinity.

Lemma 3.2. Let $\left\{\left(b_{t}, g_{t}\right)\right\}_{t \geq 0}$ be the solution to the ODE (3.1) with initial condition $\left(b_{0}, g_{0}\right) \in \Lambda$, then for any $T>0$ and $\epsilon>0$, there exist constants $C_{1}=C_{1}(T, \epsilon)$ and $N_{1}=N_{1}(T, \epsilon)$ such that

$$
P_{\lambda, n}^{n b_{0}, n g_{0}}\left(\sup _{0 \leq t \leq T}\left\|\left(\frac{B_{t}}{n}, \frac{G_{t}}{n}\right)-\left(b_{t}, g_{t}\right)\right\|_{1}>\epsilon\right) \leq \frac{C_{1}}{n}
$$

for any $n \geq N_{1}$.

Note that $C_{1}$ and $N_{1}$ do not depend on the choice of $\left(b_{0}, g_{0}\right)$.

The proof of Lemma 3.2 follows the analysis introduced by Ethier and Kurtz to construct the theory of density-dependent population model (See Chapter 11 of [4]). Readers familiar with this theory can skip the following proof.

Proof of Lemma 3.2. By the definition of $\left(b_{t}, g_{t}\right)$,

$$
\left\{\begin{array}{l}
b_{t}=b_{0}+\int_{0}^{t} F_{1}\left(b_{s}, g_{s}\right) d s, \\
g_{t}=g_{0}+\int_{0}^{t} F_{2}\left(b_{s}, g_{s}\right) d s .
\end{array}\right.
$$


Let $\left\{N_{j}(t): t \geq 0\right\}_{j=1,2,3,4}$ be four independent copies of the Poisson process with rate one, then according to the transition rates function of $\left\{B_{t}, G_{t}\right\}_{t \geq 0}$ and Theorem 6.4.1 of [4, we can write $\left(B_{t}, G_{t}\right)$ as

$$
\left\{\begin{array}{l}
B_{t}=\left\lfloor n b_{0}\right\rfloor-N_{1}\left(\int_{0}^{t} B_{s} d_{s}\right)+N_{3}\left(\int_{0}^{t} \frac{\lambda}{n} B_{s} G_{s} d s\right), \\
G_{t}=\left\lfloor n g_{0}\right\rfloor-N_{2}\left(\int_{0}^{t} G_{s} d s\right)-N_{3}\left(\int_{0}^{t} \frac{\lambda}{n} B_{s} G_{s} d s\right)+N_{4}\left(\int_{0}^{t} \frac{\lambda}{n} B_{s}\left(n-B_{s}-G_{s}\right) d s\right) .
\end{array}\right.
$$

For $j=1,2,3,4$, we define $\widetilde{N}_{j}(t)=N_{j}(t)-t$, then $\left\{\widetilde{N}_{j}(t): t \geq 0\right\}$ is a martingale with $E \widetilde{N}_{j}(t)=0$ and $E\left[\widetilde{N}_{j}^{2}(t)\right]=t$. According to Equations (3.2), (3.3) and the definition of $F=\left(F_{1}, F_{2}\right)$,

$$
\left\{\begin{aligned}
\frac{B_{t}}{n}-b_{t}= & \frac{\left\lfloor n b_{0}\right\rfloor-n b_{0}}{n}+\int_{0}^{t} F_{1}\left(\frac{B_{s}}{n}, \frac{G_{s}}{n}\right)-F_{1}\left(b_{s}, g_{s}\right) d s-\frac{\widetilde{N}_{1}\left(\int_{0}^{t} B_{s} d_{s}\right)}{n}+\frac{\widetilde{N}_{3}\left(\int_{0}^{t} \frac{\lambda}{n} B_{s} G_{s} d s\right)}{n}, \\
\frac{G_{t}}{n}-g_{t}= & \frac{\left\lfloor n g_{0}\right\rfloor-n g_{0}}{n}+\int_{0}^{t} F_{2}\left(\frac{B_{s}}{n}, \frac{G_{s}}{n}\right)-F_{2}\left(b_{s}, g_{s}\right) d s \\
& -\frac{\widetilde{N}_{2}\left(\int_{0}^{t} G_{s} d s\right)}{n}-\frac{\widetilde{N}_{3}\left(\int_{0}^{t} \frac{\lambda}{n} B_{s} G_{s} d s\right)}{n}+\frac{\widetilde{N}_{4}\left(\int_{0}^{t} \frac{\lambda}{n} B_{s}\left(n-B_{s}-G_{s}\right) d s\right)}{n} .
\end{aligned}\right.
$$

Since $B_{t} \leq n,\left|\widetilde{N}_{1}\left(\int_{0}^{t} B_{s} d_{s}\right)\right| \leq \max _{0 \leq s \leq n t}\left|\widetilde{N}_{1}(s)\right|$. For similar reasons,

$$
\begin{aligned}
\left|\widetilde{N}_{2}\left(\int_{0}^{t} B_{s} d_{s}\right)\right| & \leq \max _{0 \leq s \leq n t}\left|\widetilde{N}_{1}(s)\right|, \\
\left|\widetilde{N}_{3}\left(\int_{0}^{t} \frac{\lambda}{n} B_{s} G_{s} d s\right)\right| & \leq \max _{0 \leq s \leq \lambda n t}\left|\widetilde{N}_{3}(s)\right|, \\
\left|\widetilde{N}_{4}\left(\int_{0}^{t} \frac{\lambda}{n} B_{s} G_{s} d s\right)\right| & \leq \max _{0 \leq s \leq \lambda n t}\left|\widetilde{N}_{4}(s)\right| .
\end{aligned}
$$

It is easy to check that $F=\left(F_{1}, F_{2}\right)$ satisfies the global Lipschitz condition on $\Lambda$ under the norm $\|\cdot\|_{1}$, hence there exists $K>0$ such that

$$
\left\|F\left(b_{1}, g_{1}\right)-F\left(b_{2}, g_{2}\right)\right\|_{1} \leq K\left\|\left(b_{1}, g_{1}\right)-\left(b_{2}, g_{2}\right)\right\|_{1}
$$

for any $\left(b_{1}, g_{1}\right),\left(b_{2}, g_{2}\right) \in \Lambda$. It is obviously that $\frac{1}{n}\left(B_{t}, G_{t}\right) \in \Lambda$ for ant $t \geq 0$. As we have shown in Lemma 3.1. $\left(b_{t}, g_{t}\right) \in \Lambda$ for any $t \geq 0$. As a result, by Equation (3.4),

$$
\left\|\left(\frac{B_{t}}{n}, \frac{G_{t}}{n}\right)-\left(b_{t}, g_{t}\right)\right\|_{1} \leq \int_{0}^{t} K\left\|\left(\frac{B_{s}}{n}, \frac{G_{s}}{n}\right)-\left(b_{s}, g_{s}\right)\right\|_{1} d s+\frac{M(\lambda, n, t)}{n}
$$

for any $t \geq 0$, where

$$
M(\lambda, n, t)=2+\max _{0 \leq s \leq n t}\left|\widetilde{N}_{1}(s)\right|+\max _{0 \leq s \leq n t}\left|\widetilde{N}_{2}(s)\right|+2 \max _{0 \leq s \leq \lambda n t}\left|\widetilde{N}_{3}(s)\right|+\max _{0 \leq s \leq \lambda n t}\left|\widetilde{N}_{4}(s)\right| .
$$

Since $M(\lambda, n, t)$ increases with $t$,

$$
\left\|\left(\frac{B_{t}}{n}, \frac{G_{t}}{n}\right)-\left(b_{t}, g_{t}\right)\right\|_{1} \leq \int_{0}^{t} K\left\|\left(\frac{B_{s}}{n}, \frac{G_{s}}{n}\right)-\left(b_{s}, g_{s}\right)\right\|_{1} d s+\frac{M(\lambda, n, T)}{n}
$$

for $0 \leq t \leq T$. Then by Grownwall's inequality,

$$
\left\|\left(\frac{B_{t}}{n}, \frac{G_{t}}{n}\right)-\left(b_{t}, g_{t}\right)\right\|_{1} \leq \frac{M(\lambda, n, T)}{n} e^{K t}
$$


for $0 \leq t \leq T$. By Equation (3.6),

$$
\sup _{0 \leq t \leq T}\left\|\left(\frac{B_{t}}{n}, \frac{G_{t}}{n}\right)-\left(b_{t}, g_{t}\right)\right\|_{1} \leq \frac{M(\lambda, n, T)}{n} e^{K T} .
$$

Since $\left\{\widetilde{N}_{1}(t): t \geq 0\right\}$ is a martingale, according to Doob's inequality,

$$
P\left(\max _{0 \leq s \leq n T}\left|\widetilde{N}_{1}(s)\right| \geq n \epsilon_{0}\right) \leq \frac{1}{n^{2} \epsilon_{0}^{2}} E\left[\widetilde{N}_{1}^{2}(n T)\right]=\frac{T}{n \epsilon_{0}^{2}}
$$

for any $\epsilon_{0}>0$. For given $\epsilon>0$, we choose $\epsilon_{0}=\frac{\epsilon}{8 e^{K T}}$, then

$$
P\left(\max _{0 \leq s \leq n T}\left|\tilde{N}_{1}(s)\right| \geq \frac{n \epsilon}{8 e^{K T}}\right) \leq \frac{64 e^{2 K T} T}{n \epsilon^{2}} .
$$

According similar analysis,

$$
\begin{gathered}
P\left(\max _{0 \leq s \leq n T}\left|\widetilde{N}_{2}(s)\right| \geq \frac{n \epsilon}{8 e^{K T}}\right) \leq \frac{64 e^{2 K T} T}{n \epsilon^{2}}, \\
P\left(2 \max _{0 \leq s \leq \lambda n T}\left|\widetilde{N}_{3}(s)\right| \geq \frac{n \epsilon}{8 e^{K T}}\right) \leq \frac{256 e^{2 K T} \lambda T}{n \epsilon^{2}}, \\
P\left(\max _{0 \leq s \leq \lambda n T}\left|\widetilde{N}_{4}(s)\right| \geq \frac{n \epsilon}{8 e^{K T}}\right) \leq \frac{64 e^{2 K T} \lambda T}{n \epsilon^{2}} .
\end{gathered}
$$

Therefore,

$$
P\left(M(\lambda, n, T) \geq 2+\frac{n \epsilon}{2 e^{K T}}\right) \leq \frac{128 e^{2 K T} T+320 e^{2 K T} \lambda T}{\epsilon^{2}} .
$$

As a result,

$$
P\left(\frac{M(\lambda, n, T)}{n} \geq \frac{\epsilon}{e^{K T}}\right) \leq \frac{C_{1}(T, \epsilon)}{n}
$$

for $n \geq N_{1}(T, \epsilon)=\frac{4 e^{K T}}{\epsilon}$, where

$$
C_{1}(T, \epsilon)=\frac{128 e^{2 K T} T+320 e^{2 K T} \lambda T}{\epsilon^{2}} .
$$

Then by Equation (3.7),

$$
P_{\lambda, n}^{n, 0}\left(\sup _{0 \leq t \leq T}\left\|\left(\frac{B_{t}}{n}, \frac{G_{t}}{n}\right)-\left(b_{t}, g_{t}\right)\right\|_{1}>\epsilon\right) \leq P\left(\frac{M(\lambda, n, T)}{n} \geq \frac{\epsilon}{e^{K T}}\right) \leq \frac{C_{1}(T, \epsilon)}{n}
$$

for $n \geq N_{1}(T, \epsilon)$ and the proof is complete.

The next lemma is crucial for the proof of Equation (2.2).

Lemma 3.3. Let $\left\{\left(b_{t}, g_{t}\right)\right\}_{t \geq 0}$ be the solution to ODE (3.1) with initial condition $\left(b_{0}, g_{0}\right)=$ $(1,0)$, then when $\lambda<4$,

$$
\lim _{t \rightarrow+\infty}\left\|\left(b_{t}, g_{t}\right)\right\|_{1}=0
$$

Proof. It is easy to check that $F_{2}(b, g) \geq 0$ when and only when $g \leq \frac{\lambda b(1-b)}{2 \lambda b+1}$. Let $g^{*}=$ $\max \left\{\frac{\lambda b(1-b)}{2 \lambda b+1}: 0 \leq b \leq 1\right\}$, then it is easy to check that $g^{*}<\frac{1}{\lambda}$ when $\lambda<4$. Let $\widetilde{g}=\frac{\frac{1}{\lambda}+g^{*}}{2}$, 
then $g_{0}=0<\widetilde{g}$. For any $0 \leq b \leq 1, F_{2}(b, \widetilde{g})<0$ since $\widetilde{g}>g^{*}$. As a result, $g_{t}$ can never exceed $\widetilde{g}$ since $\left.\frac{d}{d t} g_{t}\right|_{g_{t}=\widetilde{g}}=F_{2}\left(b_{t}, \widetilde{g}\right)<0$. Therefore, $g_{t} \leq \widetilde{g}$ for any $t \geq 0$. Then,

$$
\frac{d}{d t} b_{t} \leq-b_{t}+\lambda \widetilde{g} b_{t}=(\lambda \widetilde{g}-1) b_{t}
$$

and hence

$$
b_{t} \leq e^{(\lambda \widetilde{g}-1) t}
$$

for any $t \geq 0$. By Equation (3.8),

$$
\frac{d}{d t} g_{t}=F_{2}\left(b_{t}, g_{t}\right) \leq-g_{t}+\lambda e^{(\lambda \widetilde{g}-1) t}
$$

for any $t \geq 0$. As a result,

$$
\frac{d}{d t}\left(e^{t} g_{t}\right) \leq \lambda e^{\lambda \widetilde{g} t}
$$

and hence

$$
g_{t} \leq \frac{e^{(\lambda \widetilde{g}-1) t}-e^{-t}}{\widetilde{g}} .
$$

Since $\widetilde{g}<\frac{1}{\lambda}, \lambda \widetilde{g}-1<0$. As a result, Lemma 3.3 follows from Equations (3.8) and (3.9) directly.

\section{Proof of Equation (2.1)}

In this section we give the proof of Equation (2.1). The intuitive idea of the proof is as follows. When $\lambda>4$, it is easy to check that there exists $\left(b_{0}, g_{0}\right)$ in the inner of $\Lambda$ such that $F_{1}\left(b_{0}, g_{0}\right)>0$ and $F_{2}\left(b_{0}, g_{0}\right)>0$. Then, by analyzing the vector-field of ODE (3.1), it is easy to check that the solution $\left\{\left(b_{t}, g_{t}\right)\right\}_{t \geq 0}$ with initial condition $\left(b_{0}, g_{0}\right)$ is absorbed in the area

$$
\Lambda_{1}=\left\{(b, g): b \geq b_{0}, b+g \geq b_{0}+g_{0}\right\} .
$$

As shown in Lemma 3.2 conditioned on $\left(B_{0}, G_{0}\right)=\left(n b_{0}, n g_{0}\right), \frac{1}{n}\left(B_{t}, G_{t}\right)$ is approximate to $\left(b_{t}, g_{t}\right)$. Hence $\left(B_{t}, G_{t}\right)$ should stay in the area $n \Lambda_{1}$ for a long time. Since $\left(n b_{0}, n g_{0}\right) \preceq(n, 0)$ and $\left\{\left(B_{t}, G_{t}\right)\right\}_{t \geq 0}$ is monotone under the partial order $\preceq$ by Lemma 2.2. $\left(B_{t}, G_{t}\right)$ with $\left(B_{0}, G_{0}\right)=(n, 0)$ should stay in $n \Lambda$ for a longer time.

Our proof is the effort to make the above intuitive idea rigorous and show that the precise meaning of 'long time' is $\exp \{O(n)\}$ units of time. First we show the existence of $\left(b_{0}, g_{0}\right)$. We define

$$
\widetilde{\Lambda}=\{(b, g): b>0, g>0, b+g<1\}
$$

as the inner of $\Lambda$.

Lemma 4.1. When $\lambda>4$, there exists $\left(b_{0}, g_{0}\right) \in \widetilde{\Lambda}$ such that

$$
F_{1}\left(b_{0}, g_{0}\right)>0 \text { and } F_{2}\left(b_{0}, g_{0}\right)>0
$$

where $F=\left(F_{1}, F_{2}\right)$ is as defined in $O D E$ (3.1). 
Proof. By direct calculation, when $\lambda>4,\left(\frac{\lambda-2}{2 \lambda}, \frac{1}{\lambda}\right) \in \widetilde{\Lambda}$ and

$$
F_{2}\left(\frac{\lambda-2}{2 \lambda}, \frac{1}{\lambda}\right)>0
$$

Then, we can choose sufficiently small positive $\beta$ such that $F_{2}\left(\frac{\lambda-2}{2 \lambda}, \frac{1}{\lambda}+\beta\right)>0$ and $\left(\frac{\lambda-2}{2 \lambda}, \frac{1}{\lambda}+\right.$ $\beta) \in \widetilde{\Lambda}$. For any $(b, g) \in \Lambda$ with $g>\frac{1}{\lambda}, F_{1}(b, g)>0$. As a result, Lemma 4.1 holds with

$$
\left(b_{0}, g_{0}\right)=\left(\frac{\lambda-2}{2 \lambda}, \frac{1}{\lambda}+\beta\right) .
$$

For later use, we choose $\alpha>0$ sufficiently small such that $(1-\alpha)\left(b_{0}, g_{0}\right),(1+\alpha)\left(b_{0}, g_{0}\right) \in$ $\widetilde{\Lambda}, \bar{g}, \underline{g}>0$ and

$$
\begin{aligned}
& \lambda(1-\alpha) b_{0} \underline{g}>(1+\alpha) b_{0}, \\
& \lambda(1-\alpha) b_{0}\left[1-(1+\alpha)\left(b_{0}+g_{0}\right)\right]>\bar{g}+\lambda(1+\alpha) b_{0} \bar{g},
\end{aligned}
$$

where $\underline{g}=(1-\alpha)\left(b_{0}+g_{0}\right)-(1+\alpha) b_{0}$ and $\bar{g}=(1+\alpha)\left(b_{0}+g_{0}\right)-(1-\alpha) b_{0}$. Note that the existence of $\alpha$ depends on the fact that

$$
\begin{aligned}
& \lambda b_{0} g_{0}>b_{0}, \\
& \lambda b_{0}\left[1-\left(b_{0}+g_{0}\right)\right]>g_{0}+\lambda b_{0} g_{0} .
\end{aligned}
$$

according to Lemma 4.1

We define

$$
\Lambda_{2}=\left\{(b, g):(1-\alpha) b_{0} \leq b \leq(1+\alpha) b_{0},(1-\alpha)\left(b_{0}+g_{0}\right) \leq b+g \leq(1+\alpha)\left(b_{0}+g_{0}\right)\right\},
$$

then it is easy to check that

$$
\underline{g}=\min \left\{g:(b, g) \in \Lambda_{2}\right\} \text { and } \bar{g}=\max \left\{g:(b, g) \in \Lambda_{2}\right\} .
$$

We define

$$
\gamma=\inf \left\{t \geq 0: \frac{1}{n}\left(B_{t}, G_{t}\right) \notin \Lambda_{2}\right\}
$$

as the first moment that $(B, G)$ exits $n \Lambda_{2}$. Furthermore, we define

$$
D=\inf \left\{\left\|(b, g)-\left(b_{0}, g_{0}\right)\right\|_{1}:(b, g) \notin \Lambda_{2}\right\}>0 .
$$

The next lemma about the time $(B, G)$ waits for to exit $n \Lambda_{2}$ conditioned on $\left(B_{0}, G_{0}\right)=$ $\left(n b_{0}, n g_{0}\right)$ is utilized later.

Lemma 4.2. For given $\lambda>4$ and $\alpha$ defined as in Equation (4.1), there exists $C_{4}=$ $C_{4}(\lambda, \alpha)>0$ and $N_{6}=N_{6}(\lambda, \alpha)>0$ such that

$$
P_{\lambda, n}^{n b_{0}, n g_{0}}\left(\gamma \geq \frac{D}{4(1+\lambda)}\right) \geq 1-e^{-C_{4} n}
$$

for $n \geq N_{6}$. 
Proof. Conditioned on $\left(B_{0}, G_{0}\right)=\left(n b_{0}, n g_{0}\right)$, the $l_{1}$ norm $\|(B, G)\|_{1}$ must change by at least $n D$ for $\frac{1}{n}(B, G)$ to exit $\Lambda_{2}$. At each moment that $(B, G)$ jumps, $\|(B, G)\|_{1}$ changes by at most 2. Hence $(B, G)$ must jump at least $\frac{n D}{2}$ times to exit $n \Lambda_{2}$. It is easy to check that $(B, G)$ changes state with rate at most

$$
n+\frac{\lambda}{n} n n=(1+\lambda) n
$$

As a result,

$$
P_{\lambda, n}^{n b_{0}, n g_{0}}\left(\gamma \leq \frac{D}{4(1+\lambda)}\right) \leq P\left(Y_{n}\left(\frac{D}{4(1+\lambda)}\right) \geq \frac{n D}{2}\right),
$$

where $\left\{Y_{n}(t)\right\}_{t \geq 0}$ is the Poisson process with rate $(1+\lambda) n$ for each $n \geq 1$. $Y_{n}(t)$ has the same probability distribution as that of $Y_{1}(n t)$, hence

$$
P_{\lambda, n}^{n b_{0}, n g_{0}}\left(\gamma \leq \frac{D}{4(1+\lambda)}\right) \leq P\left(\frac{Y_{1}\left(n \frac{D}{4(1+\lambda)}\right)}{n} \geq \frac{D}{2}\right)
$$

According to classic limit theorems of the Poisson process, for any $t>0, Y_{1}(n t) / n$ converges to $(1+\lambda) t$ in probability as $n \rightarrow+\infty$ and there exists $I(t)>0$ such that

$$
P\left(\frac{Y_{1}(n t)}{n} \geq 2(1+\lambda) t\right) \leq e^{-n I(t)}
$$

for sufficiently large $n$. As a result, there exists $N_{6}=N_{6}(\lambda, \alpha)$ such that

$$
P\left(\frac{Y_{1}\left(n \frac{D}{4(1+\lambda)}\right)}{n} \geq \frac{D}{2}\right) \leq e^{-n I\left(\frac{D}{4(1+\lambda)}\right)}
$$

for $n \geq N_{6}$. Lemma 4.2 follows from Equations (4.3) and (4.4) directly with $C_{4}=I\left(\frac{D}{4(1+\lambda)}\right)$.

We introduce a birth-and-death process as an auxiliary model for the proof of Equation (2.1). Let $\left\{\left(\widehat{B}_{t}, \widehat{S}_{t}\right)\right\}_{t \geq 0}$ be the birth-and-death process with transition rates function given by

$$
(\widehat{B}, \widehat{S}) \rightarrow \begin{cases}(\widehat{B}, \widehat{S})-(1,1) & \text { at rate } n(1+\alpha) b_{0} \\ (\widehat{B}, \widehat{S})-(0,1) & \text { at rate } n \bar{g} \\ (\widehat{B}, \widehat{S})+(1,0) & \text { at rate } \lambda n(1-\alpha) b_{0} \underline{\underline{g}} \\ (\widehat{B}, \widehat{S})+(0,1) & \text { at rate } \lambda n(1-\alpha) b_{0}\left[1-(1+\alpha)\left(b_{0}+g_{0}\right)\right]\end{cases}
$$

Later we will show that $B_{t} \geq \widehat{B}_{t}$ and $B_{t}+G_{t} \geq \widehat{S}_{t}$ in the sense of coupling for $t \in[0, \gamma]$, for which we introduce $(\widehat{B}, \widehat{S})$. The next lemma shows that for any $t>0, \widehat{S}_{t} \geq \widehat{S}_{0}$ and $\widehat{B}_{t} \geq \widehat{B}_{0}$ with high probability.

Lemma 4.3. There exists $C_{5}=C_{5}(\lambda, \alpha)>0$ such that for any $t>0$ and $n \geq 1$,

$$
P\left(\widehat{B}_{t} \geq n b_{0}, \widehat{S}_{t} \geq n g_{0}+n b_{0} \mid\left(\widehat{B}_{0}, \widehat{S}_{0}\right)=\left(n b_{0}, n g_{0}+n b_{0}\right)\right) \geq 1-2 e^{-C_{5} n t} .
$$

Proof. Throughout this proof we assume that $\left(\widehat{B}_{0}, \widehat{S}_{0}\right)=\left(n b_{0}, n b_{0}+n g_{0}\right)$. Let $q_{1}=\lambda(1-$ $\alpha) b_{0}\left[1-(1+\alpha)\left(b_{0}+g_{0}\right)\right]$ and $q_{2}=(1+\alpha) b_{0}+\bar{g}$, then $\left\{\widehat{S}_{t}\right\}_{t \geq 0}$ is a birth-and-death process with transition rates function given by

$$
\widehat{S} \rightarrow \begin{cases}\widehat{S}+1 & \text { at rate } n q_{1}, \\ \widehat{S}-1 & \text { at rate } n q_{2} .\end{cases}
$$


By Equation (4.1), it is easy to check that $q_{1}>q_{2}$, then we can choose $\frac{q_{2}}{q_{1}}<\rho<1$. Since $\rho<1$, by Chebyshev's inequality,

$$
P\left(\widehat{S}_{t} \leq n b_{0}+n g_{0}\right)=P\left(\rho^{\widehat{S}_{t}} \geq \rho^{n b_{0}+n g_{0}}\right) \leq \rho^{-n b_{0}-n g_{0}} E \rho^{\widehat{S}_{t}} .
$$

According to the transition rates function of $\widehat{S}$,

$$
\begin{aligned}
\frac{d}{d t} E \rho^{\widehat{S}_{t}} & =n q_{1}\left(E \rho^{\widehat{S}_{t}+1}-E \rho^{\widehat{S}_{t}}\right)+n q_{2}\left(E \rho^{\widehat{S}_{t}-1}-E \rho^{\widehat{S}_{t}}\right) \\
& =n\left(q_{1} \rho+\frac{q_{2}}{\rho}-q_{1}-q_{2}\right) E \rho^{\widehat{S}_{t}} .
\end{aligned}
$$

Then,

$$
E \rho^{\widehat{S}_{t}}=\rho^{n b_{0}+n g_{0}} e^{-C_{6} n t}
$$

since $\widehat{S}_{0}=n b_{0}+n g_{0}$, where $C_{6}=q_{1}+q_{2}-q_{1} \rho-\frac{q_{2}}{\rho}$. Note that $C_{6}>0$ since $\frac{q_{2}}{q_{1}}<\rho<1$. By Equations (4.5) and (4.6),

$$
P\left(\widehat{S}_{t} \leq n b_{0}+n g_{0}\right) \leq e^{-C_{6} n t} .
$$

According to the same analysis as that gives Equation (4.7), there exists $C_{7}>0$ such that

$$
P\left(\widehat{B}_{t} \leq n b_{0}\right) \leq e^{-C_{7} n t} .
$$

Let $C_{5}=\min \left\{C_{6}, C_{7}\right\}$, then Lemma 4.3 follows from Equations (4.7) and (4.8) directly.

The next lemma shows that $B_{t}$ and $B_{t}+G_{t}$ are bounded from below by $\widehat{B}_{t}$ and $\widehat{S}_{t}$ respectively for $t \geq[0, \gamma]$.

Lemma 4.4. Conditioned on $\left(B_{0}, B_{0}+G_{0}\right)=\left(\widehat{B}_{0}, \widehat{S}_{0}\right)=\left(n b_{0}, n b_{0}+n g_{0}\right)$,

$$
B_{t} \geq \widehat{B}_{t} \text { and } B_{t}+G_{t} \geq \widehat{S}_{t}
$$

in the sense of coupling for $t \in[0, \gamma]$.

Proof. For $t \in[0, \gamma], \frac{1}{n}\left(B_{t}, G_{t}\right) \in \Lambda_{2}$. The transition rates function of $\left\{\left(B_{t}, B_{t}+G_{t}\right)\right\}_{t \geq 0}$ is given by

$$
(B, B+G) \rightarrow \begin{cases}(B, B+G)-(1,1) & \text { at rate } F_{1}(B, G)=B, \\ (B, B+G)-(0,1) & \text { at rate } F_{2}(B, G)=G, \\ (B, B+G)+(1,0) & \text { at rate } F_{3}(B, G)=\frac{\lambda}{n} B G, \\ (B, B+G)+(0,1) & \text { at rate } F_{4}(B, G)=\frac{\lambda}{n} B(n-B-G) .\end{cases}
$$

According to the definition of $\Lambda_{2}$, for any $(B, G)$ that $\frac{1}{n}(B, G) \in \Lambda_{2}$,

$$
\begin{aligned}
& F_{1}(B, G) \leq n(1+\alpha) b_{0}, \quad F_{2}(B, G) \leq n \bar{g}, \\
& F_{3}(B, G) \geq \lambda n(1-\alpha) b_{0} \underline{g}, \quad F_{4}(B, G) \geq \lambda n(1-\alpha) b_{0}\left[1-(1+\alpha)\left(b_{0}+g_{0}\right)\right] .
\end{aligned}
$$


As a result, we can couple $\left(B_{t}, B_{t}+G_{t}\right)$ and $\left(\widehat{B}_{t}, \widehat{S}_{t}\right)$ as follows for $t \in[0, \gamma]$.

$$
\begin{aligned}
& (B, B+G, \widehat{B}, \widehat{S}) \\
& \quad \begin{cases}(B, B+G, \widehat{B}, \widehat{S})-(1,1,1,1) & \text { at rate } F_{1}(B, G), \\
(B, B+G, \widehat{B}, \widehat{S})-(0,0,1,1) & \text { at rate } n(1+\alpha) b_{0}-F_{1}(B, G), \\
(B, B+G, \widehat{B}, \widehat{S})-(0,1,0,1) & \text { at rate } F_{2}(B, G), \\
(B, B+G, \widehat{B}, \widehat{S})-(0,0,0,1) & \text { at rate } n \bar{g}-F_{2}(B, G), \\
(B, B+G, \widehat{B}, \widehat{S})+(1,0,1,0) & \text { at rate } \lambda n(1-\alpha) b_{0} \underline{g}, \\
(B, B+G, \widehat{B}, \widehat{S})+(1,0,0,0) & \text { at rate } F_{3}(B, G)-\lambda n(1-\alpha) b_{0} \underline{g}, \\
(B, B+G, \widehat{B}, \widehat{S})+(0,1,0,1) & \text { at rate } \lambda n(1-\alpha) b_{0}\left[1-(1+\alpha)\left(b_{0}+g_{0}\right)\right], \\
(B, B+G, \widehat{B}, \widehat{S})+(0,1,0,0) & \text { at rate } F_{4}(B, G)-\lambda n(1-\alpha) b_{0}\left[1-(1+\alpha)\left(b_{0}+g_{0}\right)\right] .\end{cases}
\end{aligned}
$$

The above coupling does not change the property that $B \geq \widehat{B}$ and $B+G \geq \widehat{S}$, hence the proof is complete.

At last we give the proof of Equation (2.1).

Proof of Equation (2.1). We define

$$
\Lambda_{1}=\left\{(b, g) \in \Lambda: b \geq b_{0}, b+g \geq b_{0}+g_{0}\right\}
$$

as we have done at the beginning of this section.

Let $T_{4}=\frac{D}{4(1+\lambda)}>0$, where $D$ is defined as in Lemma 4.2 then the first step of this proof is to show that

$$
P_{\lambda, n}^{n b_{0}, n g_{0}}\left(\frac{\left(B_{T_{4}}, G_{T_{4}}\right)}{n} \in \Lambda_{1}\right) \geq 1-3 e^{-C_{8} n}
$$

for some $C_{8}=C_{8}(\lambda)>0$ and sufficiently large $n$. The proof of Equation (4.9) is as follows. By Lemma 4.4 $B_{t} \geq \widehat{B}_{t}$ and $B_{t}+G_{t} \geq \widehat{S}_{t}$ for $t \in[0, \gamma]$, where $\left(B_{0}, G_{0}+B_{0}\right)=\left(\widehat{B}_{0}, \widehat{S}_{0}\right)=$ $\left(n b_{0}, n b_{0}+n g_{0}\right)$. Therefore,

$$
\begin{aligned}
P_{\lambda, n}^{n b_{0}, n g_{0}}\left(\frac{\left(B_{T_{4}}, G_{T_{4}}\right)}{n} \in \Lambda_{1}\right) & \geq P_{\lambda, n}^{n b_{0}, n g_{0}}\left(\frac{\left(B_{T_{4}}, G_{T_{4}}\right)}{n} \in \Lambda_{1}, \gamma \geq T_{4}\right) \\
& =P_{\lambda, n}^{n b_{0}, n g_{0}}\left(B_{T_{4}} \geq n b_{0}, B_{T_{4}}+G_{T_{4}} \geq n b_{0}+n g_{0}, \gamma \geq T_{4}\right) \\
& \geq P_{\lambda, n}^{n b_{0}, n g_{0}}\left(\widehat{B}_{T_{4}} \geq n b_{0}, \widehat{S}_{T_{4}} \geq n b_{0}+n g_{0}, \gamma \geq T_{4}\right) \\
& \geq P\left(\widehat{B}_{T_{4}} \geq n b_{0}, \widehat{S}_{T_{4}} \geq n b_{0}+n g_{0}\right)-P_{\lambda, n}^{n b_{0}, n g_{0}}\left(\gamma<T_{4}\right) \\
& \geq 1-2 e^{-C_{5} T_{4} n}-e^{-C_{4} n}
\end{aligned}
$$

for $n \geq N_{6}$, where $N_{6}, C_{4}$ are defined as in Lemma 4.2 while $C_{5}$ is defined as in Lemma 4.3. Note that the last inequality in Equation (4.10) follows from Lemmas 4.2 and 4.3 . As a result, Equation (4.9) holds with $C_{8}=\min \left\{C_{5} T_{4}, C_{4}\right\}$ and $n \geq N_{6}$.

Note that if $\left(B_{1}, G_{1}\right) \succeq\left(B_{2}, G_{2}\right)$ and $\frac{1}{n}\left(B_{2}, G_{2}\right) \in \Lambda_{1}$, then $\frac{1}{n}\left(B_{1}, G_{1}\right) \in \Lambda_{1}$. Therefore, by Lemma 2.2,

$$
P_{\lambda, n}^{n b_{0}, n g_{0}}\left(\frac{\left(B_{T_{4}}, G_{T_{4}}\right)}{n} \in \Lambda_{1}\right) \leq P_{\lambda, n}^{B, G}\left(\frac{\left(B_{T_{4}}, G_{T_{4}}\right)}{n} \in \Lambda_{1}\right)
$$


for any $(B, G) \succeq\left(n b_{0}, n g_{0}\right)$. Then by Equation (4.9),

$$
P_{\lambda, n}^{B, G}\left(\left(B_{T_{4}}, G_{T_{4}}\right) \succeq\left(n b_{0}, n g_{0}\right)\right)=P_{\lambda, n}^{B, G}\left(\frac{\left(B_{T_{4}}, G_{T_{4}}\right)}{n} \in \Lambda_{1}\right) \geq 1-3 e^{-C_{8} n}
$$

for any $(B, G) \succeq\left(n b_{0}, n g_{0}\right)$ and $n \geq N_{6}$. By Equation (4.11), utilizing the Markov property for $e^{\frac{C_{8}}{2} n}$ times,

$$
P_{\lambda, n}^{n b_{0}, n g_{0}}\left(\left(B_{T_{4} e^{\frac{C_{8}}{2} n}}, G_{T_{4} e^{\frac{C_{8}}{2} n}}\right) \succeq\left(n b_{0}, n g_{0}\right)\right) \geq\left(1-3 e^{-C_{8} n}\right)^{e^{\frac{C_{8}}{2} n}} \geq 1-3 e^{-\frac{C_{8}}{2} n}
$$

for $n \geq N_{6}$ and hence

$$
P_{\lambda, n}^{n b_{0}, n g_{0}}\left(\tau>T_{4} e^{\frac{C_{8}}{2} n}\right) \geq 1-3 e^{-\frac{C_{8}}{2} n}
$$

for $n \geq N_{6}$. By Equation (4.12),

$$
P_{\lambda, n}^{n b_{0}, n g_{0}}\left(\tau>e^{\frac{C_{8}}{4} n}\right) \geq 1-3 e^{-\frac{C_{8}}{2} n}
$$

for sufficiently large $n$. Then, by Lemma 2.2 and the fact that $(n, 0) \succeq\left(n b_{0}, n g_{0}\right)$,

$$
P_{\lambda, n}^{n, 0}\left(\tau>e^{\frac{C_{8}}{4} n}\right) \geq P_{\lambda, n}^{n b_{0}, n g_{0}}\left(\tau>e^{\frac{C_{8}}{4} n}\right) \geq 1-3 e^{-\frac{C_{8}}{2} n}
$$

for sufficiently large $n$ and hence Equation (2.1) holds with $C(\lambda)=\frac{C_{8}}{4}$.

\section{$5 \quad$ Proof of Equation (2.2)}

In this section we give the proof of Equation (2.2). It is obviously that we only need to deal with small $\theta$, so we assume that $\theta<1$. Throughout this section we use $\left\{\left(b_{t}, g_{t}\right)\right\}_{t \geq 0}$ to denote the solution to ODE (3.1) with initial condition $\left(b_{0}, g_{0}\right)=(1,0)$. Sometimes we write $b_{t}, g_{t}$ as $b(t), g(t)$ when the subscript is complex. First we show that $\frac{1}{n}\left\|\left(B_{t}, G_{t}\right)\right\|_{1}$ stays small for $O(\sqrt{n})$ units of time with high probability when $\lambda<4$.

Lemma 5.1. For given $\lambda<4$ and $\theta \in(0,1)$, there exist $T_{1}=T_{1}(\lambda, \theta), N_{2}=N_{2}(\lambda, \theta)$, $C_{2}=C_{2}(\lambda, \theta)$ and $C_{3}=C_{3}(\lambda, \theta)$ such that

$$
P_{\lambda, n}^{n, 0}\left(\sup _{0 \leq t \leq C_{2} \sqrt{n}} \frac{\left\|\left(B_{T_{1}+t}, G_{T_{1}+t}\right)\right\|_{1}}{n} \geq \frac{\theta}{(3+\theta) \lambda}\right) \leq \frac{C_{3}}{\sqrt{n}}
$$

for each $n \geq N_{2}$.

Proof. By Lemma 3.3. for $\lambda<4$, we can choose $0<T_{3}(\lambda, \theta)<T_{1}(\lambda, \theta)$ such that

$$
b\left(T_{3}\right)+g\left(T_{3}\right) \leq \frac{\theta}{2(3+\theta) \lambda}, b\left(T_{1}\right)+g\left(T_{1}\right) \leq \frac{b\left(T_{3}\right)}{2}
$$

and

$$
b_{t}+g_{t} \leq \frac{\theta}{2(3+\theta) \lambda}
$$


for any $t \in\left[T_{3}, T_{1}\right]$. Let $\left\{\left(\widehat{b}_{t}, \widehat{g}_{t}\right)\right\}_{t \geq 0}$ be the solution to ODE (3.1) with $\left(\widehat{b}_{0}, \widehat{g}_{0}\right)=\left(b\left(T_{3}\right), g\left(T_{3}\right)\right)$, then $\left(\widehat{b}_{t}, \widehat{g}_{t}\right)=\left(b\left(T_{3}+t\right), g\left(T_{3}+t\right)\right)$ and hence

$$
\widehat{b}_{t}+\widehat{g}_{t} \leq \frac{\theta}{2(3+\theta) \lambda}
$$

for $t \in\left[0, T_{1}-T_{3}\right]$. Since $\frac{d}{d t} b_{t} \geq-b_{t}, b_{t} \geq b_{0} e^{-t}$ and hence $b\left(T_{3}\right)>0$. Let $T=T_{1}-T_{3}$ and $\epsilon_{1}=\min \left\{\frac{\theta}{5(3+\theta) \lambda}, \frac{b\left(T_{3}\right)}{10}\right\}>0$, then according to Lemma 3.2 .

$$
P_{\lambda, n}^{n b\left(T_{3}\right), n g\left(T_{3}\right)}\left(\sup _{0 \leq t \leq T}\left\|\frac{\left(B_{t}, G_{t}\right)}{n}-\left(\widehat{b}_{t}, \widehat{g}_{t}\right)\right\|_{1}>\epsilon_{1}\right) \leq \frac{C_{1}\left(T_{1}-T_{3}, \epsilon_{1}\right)}{n}
$$

for $n \geq N_{1}\left(T_{1}-T_{3}, \epsilon_{1}\right)$, where $C_{1}$ and $N_{1}$ are as defined in Lemma 3.2 By Equations (5.1) and (5.2),

$$
\begin{aligned}
& P_{\lambda, n}^{n b\left(T_{3}\right), n g\left(T_{3}\right)}\left(\sup _{0 \leq t \leq T_{1}-T_{3}} \frac{\left\|\left(B_{t}, G_{t}\right)\right\|_{1}}{n} \leq \frac{7 \theta}{10(3+\theta) \lambda}\right. \\
& \text { and } \left.\left(n b\left(T_{3}\right), n g\left(T_{3}\right)\right) \succeq\left(B\left(T_{1}-T_{3}\right), G\left(T_{1}-T_{3}\right)\right)\right) \geq 1-\frac{C_{1}\left(T_{1}-T_{3}, \epsilon_{1}\right)}{n}
\end{aligned}
$$

for $n \geq N_{1}\left(T_{1}-T_{3}, \epsilon_{1}\right)$. By Lemma 2.2 and Equation (5.3), for any $(B, G) \preceq\left(n b\left(T_{3}\right), n g\left(T_{3}\right)\right)$,

$$
\begin{aligned}
& P_{\lambda, n}^{B, G}\left(\sup _{0 \leq t \leq T_{1}-T_{3}} \frac{\left\|\left(B_{t}, G_{t}\right)\right\|_{1}}{n} \leq \frac{7 \theta}{10(3+\theta) \lambda}\right. \\
& \text { and } \left.\left(n b\left(T_{3}\right), n g\left(T_{3}\right)\right) \succeq\left(B\left(T_{1}-T_{3}\right), G\left(T_{1}-T_{3}\right)\right)\right) \geq 1-\frac{C_{1}\left(T_{1}-T_{3}, \epsilon_{1}\right)}{n}
\end{aligned}
$$

for $n \geq N_{1}\left(T_{1}-T_{3}, \epsilon_{1}\right)$. By Equation (5.4) and utilizing the Markov property for $\sqrt{n}$ times,

$$
\begin{aligned}
P_{\lambda, n}^{n b\left(T_{3}\right), n g\left(T_{3}\right)}\left(\sup _{0 \leq t \leq\left(T_{1}-T_{3}\right) \sqrt{N}} \frac{\left\|\left(B_{t}, G_{t}\right)\right\|_{1}}{n} \leq \frac{7 \theta}{10(\theta+3) \lambda}\right) & \geq\left(1-\frac{C_{1}\left(T_{1}-T_{3}, \epsilon_{1}\right)}{n}\right)^{\sqrt{n}} \\
& \geq 1-\frac{C_{1}\left(T_{1}-T_{3}, \epsilon_{1}\right)}{\sqrt{n}}
\end{aligned}
$$

for $n \geq N_{1}\left(T_{1}-T_{3}, \epsilon_{1}\right)$. By Lemma 3.2

$$
P_{\lambda, n}^{n, 0}\left(\left\|\frac{\left(B\left(T_{1}\right), G\left(T_{1}\right)\right)}{n}-\left(b\left(T_{1}\right), g\left(T_{1}\right)\right)\right\|_{1} \leq \frac{b\left(T_{3}\right)}{10}\right) \geq 1-\frac{C_{1}\left(T_{1}, \frac{b\left(T_{3}\right)}{10}\right)}{n}
$$

for $n \geq N_{1}\left(T_{1}, \frac{b\left(T_{3}\right)}{10}\right)$ and hence

$$
P_{\lambda, n}^{n, 0}\left(\left(B\left(T_{1}\right), G\left(T_{1}\right)\right) \preceq\left(n b\left(T_{3}\right), n g\left(T_{3}\right)\right)\right) \geq 1-\frac{C_{1}\left(T_{1}, \frac{b\left(T_{3}\right)}{10}\right)}{n}
$$

for $n \geq N_{1}\left(T_{1}, \frac{b\left(T_{3}\right)}{10}\right)$. Conditioned on $\left(B\left(T_{1}\right), G\left(T_{1}\right)\right) \preceq\left(n b\left(T_{3}\right), n g\left(T_{3}\right)\right)$, according to Lemma 2.2 and Equation (5.5),

$$
\sup _{0 \leq t \leq\left(T_{1}-T_{3}\right) \sqrt{N}} \frac{\left\|\left(B_{T_{1}+t}, G_{T_{1}+t}\right)\right\|_{1}}{n} \leq \frac{7 \theta}{10(3+\theta) \lambda}
$$


with probability at least $1-\frac{C_{1}\left(T_{1}-T_{3}, \epsilon_{1}\right)}{\sqrt{n}}$ for $n \geq N_{1}\left(T_{1}-T_{3}, \epsilon_{1}\right)$. Hence by Equation (5.6),

$$
\sup _{0 \leq t \leq\left(T_{1}-T_{3}\right) \sqrt{N}} \frac{\left\|\left(B_{T_{1}+t}, G_{T_{1}+t}\right)\right\|_{1}}{n} \leq \frac{7 \theta}{10(3+\theta) \lambda}<\frac{\theta}{(3+\theta) \lambda}
$$

with probability at least $1-\frac{C_{1}\left(T_{1}-T_{3}, \epsilon_{1}\right)}{\sqrt{n}}-\frac{C_{1}\left(T_{1}, \frac{b\left(T_{3}\right)}{10}\right)}{n}$ for $n \geq \max \left\{N_{1}\left(T_{1}, \frac{b\left(T_{3}\right)}{10}\right), N_{1}\left(T_{1}-\right.\right.$ $\left.\left.T_{3}, \epsilon_{1}\right)\right\}$. We choose $N_{3}$ such that

$$
\frac{C_{1}\left(T_{1}, \frac{b\left(T_{3}\right)}{10}\right)}{n} \leq \frac{1}{\sqrt{n}}
$$

for $n \geq N_{3}$, then Lemma 5.1 holds with $T_{1}=T_{1}(\lambda, \theta), N_{2}=\max \left\{N_{3}, N_{1}\left(T_{1}, \frac{b\left(T_{3}\right)}{10}\right), N_{1}\left(T_{1}-\right.\right.$ $\left.\left.T_{3}, \epsilon_{1}\right)\right\}, C_{2}=T_{1}-T_{3}$ and $C_{3}=1+C_{1}\left(T_{1}-T_{3}, \epsilon_{1}\right)$.

To prove Equation (2.2), we introduce the following Markov process as an auxiliary model.

Let $\left\{\widetilde{B}_{t}\right\}_{t \geq 0}$ be a continuous-time Markov process with state space $\{0,1,2, \ldots\}$ and transition rates function given by

$$
\widetilde{B} \rightarrow \begin{cases}\widetilde{B}-1 & \text { at rate } \widetilde{B}, \\ \widetilde{B}+1 & \text { at rate } \frac{\theta \widetilde{B}}{\theta+3},\end{cases}
$$

then the following lemma shows that $\widetilde{B}$ dies out in $O(\log n)$ units of time with high probability conditioned on $\widetilde{B}_{0}=n$.

Lemma 5.2.

$$
P\left(\widetilde{B}_{\left(1+\frac{\theta}{2}\right) \log n}=0 \mid \widetilde{B}_{0}=n\right) \geq 1-n^{-\frac{\theta}{2 \theta+6}}
$$

for each $n \geq 1$.

Proof. Let $h(t)=E\left(\widetilde{B}_{t} \mid \widetilde{B}_{0}=n\right)$, then according to the transition rates function of $\widetilde{B}$,

$$
\frac{d}{d t} h(t)=-h(t)+\frac{\theta h(t)}{\theta+3}=-\frac{3 h(t)}{\theta+3} .
$$

Then, $h(t)=n e^{-\frac{3 t}{\theta+3}}$ since $h(0)=n$. By Chebyshev's inequality,

$$
P\left(\widetilde{B}_{\left(1+\frac{\theta}{2}\right) \log n} \geq 1 \mid \widetilde{B}_{0}=n\right) \leq h\left(\left(1+\frac{\theta}{2}\right) \log n\right)=n^{-\frac{\theta}{2 \theta+6}} .
$$

Therefore,

$$
P\left(\widetilde{B}_{\left(1+\frac{\theta}{2}\right) \log n}=0 \mid \widetilde{B}_{0}=n\right)=1-P\left(\widetilde{B}_{\left(1+\frac{\theta}{2}\right) \log n} \geq 1 \mid \widetilde{B}_{0}=n\right) \geq 1-n^{-\frac{\theta}{2 \theta+6}} .
$$

At last we give the proof of Equation (2.2). From now on we assume that $\widetilde{B}_{0}=n$.

Proof of Equation (2.2). Let $T_{1}$ be as defined in Lemma 5.1. On the event $\left\|\left(B_{T_{1}}, G_{T_{1}}\right)\right\|_{1}<$ $\frac{n \theta}{(\theta+3) \lambda}$, we define

$$
\sigma=\inf \left\{t \geq 0:\left\|\left(B_{T_{1}+t}, G_{T_{1}+t}\right)\right\|_{1} \geq \frac{n \theta}{(\theta+3) \lambda}\right\}
$$


For $t \in\left[T_{1}, T_{1}+\sigma\right], B \rightarrow B+1$ at rate

$$
\frac{\lambda}{n} B G \leq \frac{\lambda}{n} B \frac{n \theta}{(\theta+3) \lambda}=\frac{\theta B}{\theta+3} .
$$

Therefore, conditioned on $\left\|\left(B_{T_{1}}, G_{T_{1}}\right)\right\|_{1}<\frac{n \theta}{(\theta+3) \lambda}$,

$$
B_{T_{1}+t} \leq \widetilde{B}_{t}
$$

for $t \in[0, \sigma]$ in the sense of coupling. As a result,

$$
\begin{aligned}
& P_{\lambda, n}^{n, 0}\left(B_{T_{1}+\left(1+\frac{\theta}{2}\right) \log n}=0,\left\|\left(B_{T_{1}}, G_{T_{1}}\right)\right\|_{1}<\frac{n \theta}{(\theta+3) \lambda}, \sigma>\left(1+\frac{\theta}{2}\right) \log n\right) \\
& \geq P_{\lambda, n}^{n, 0}\left(\widetilde{B}_{\left(1+\frac{\theta}{2}\right) \log n}=0,\left\|\left(B_{T_{1}}, G_{T_{1}}\right)\right\|_{1}<\frac{n \theta}{(\theta+3) \lambda}, \sigma>\left(1+\frac{\theta}{2}\right) \log n\right) \\
& \geq P\left(\widetilde{B}_{\left(1+\frac{\theta}{2}\right) \log n}=0\right)-P_{\lambda, n}^{n, 0}\left(\sup _{0 \leq t \leq\left(1+\frac{\theta}{2}\right) \log n}\left\|\left(B_{T_{1}+t}, G_{T_{1}+t}\right)\right\|_{1} \geq \frac{n \theta}{(\theta+3) \lambda}\right) .
\end{aligned}
$$

We choose $N_{4}$ such that $\left(1+\frac{\theta}{2}\right) \log n \leq C_{2} \sqrt{n}$ for each $n \geq N_{4}$, then by Lemma 5.1,

$$
P_{\lambda, n}^{n, 0}\left(\sup _{0 \leq t \leq\left(1+\frac{\theta}{2}\right) \log n}\left\|\left(B_{T_{1}+t}, G_{T_{1}+t}\right)\right\|_{1} \geq \frac{n \theta}{(\theta+3) \lambda}\right) \leq \frac{C_{3}}{\sqrt{n}}
$$

for $n \geq N_{5}=\max \left\{N_{4}, N_{2}\right\}$. By Equations (5.7), (5.8) and Lemma 5.2

$$
P_{\lambda, n}^{n, 0}\left(B_{T_{1}+\left(1+\frac{\theta}{2}\right) \log n}=0\right) \geq 1-\frac{C_{3}}{\sqrt{n}}-n^{-\frac{\theta}{2 \theta+6}}
$$

for $n \geq N_{5}$. By Equation (5.9),

$$
P_{\lambda, n}^{n, 0}\left(\tau \leq T_{1}+\left(1+\frac{\theta}{2}\right) \log n\right) \geq 1-\frac{C_{3}}{\sqrt{n}}-n^{-\frac{\theta}{2 \theta+6}}
$$

for $n \geq N_{5}$. Equation (2.2) follows from Equation (5.10) directly since

$$
T_{1}+\left(1+\frac{\theta}{2}\right) \log n \leq(1+\theta) \log n
$$

for sufficiently large $n$.

Acknowledgments. The author is grateful to Dr. Zhichao Shan, who suggests us to study the contact process with semi-infected state. The author is grateful to the financial support from the National Natural Science Foundation of China with grant number 11501542 and the financial support from Beijing Jiaotong University with grant number KSRC16006536. 


\section{References}

[1] Durrett, R. and Liu X. (1988). The contact process on a finite set. The Annals of Probability 16, 1158-1173.

[2] Durrett, R. and Schonmann, R. H. (1988). The contact process on a finite set II. The Annals of Probability 16, 1570-1583.

[3] Durrett, R., Schonmann, R. H. and Tanaka, N. (1989). The contact process on a finite set III. The Annals of Probability 17, 1303-1321.

[4] Ethier, N. and Kurtz, T. (1986). Markov Processes: Characterization and Convergence. John Wiley and Sons, Hoboken, NJ, USA.

[5] Foxall, E., Edwards, R. and van den Driessche, P. (2016). Social contact processes and the partner model. The Annals of Applied Probability 26, 1297-1328.

[6] Harris, T. E. (1974). Contact interactions on a lattice. The Annals of Probability 2, 969-988.

[7] Liggett, T. M. (1985). Interacting Particle Systems. Springer, New York.

[8] Liggett, T. M. (1999). Stochastic interacting systems: contact, voter and exclusion processes. Springer, New York.

[9] Peterson, J. (2011). The contact process on the complete graph with random vertexdependent infection rates. Stochastic Processes and their Applications 121(3), 609-629. 